\title{
Demand analysis on the performance of shipboard alert unmanned aerial vehicles
}

\author{
Shufan Zhao ${ }^{1, *}$, Liming $\mathrm{Shi}^{2}$, Qingbo $\mathrm{Qu}^{3}$, Feixiang Zhu ${ }^{1}$, and Xinyi Yang ${ }^{1}$ \\ ${ }^{1}$ Aviation Foundation College, Naval Aviation University, Yantai Shandong 264001, China \\ ${ }^{2}$ Unit 92198, Huludao, Liaoning 125109, China \\ ${ }^{3}$ Unit 91911, Sanya, Hainan 572000, China
}

\begin{abstract}
Shipboard alert unmanned aerial vehicles (UAV) has great significance to improve early warning detection capability of aircraft carrier formation. This paper first analyzes and summarizes the tactical and technical indicators of the carrier-based air early warning(AEW), then analyzes the tactical and technical indicators of the UAV refer to technical performance of early warning aircraft, and finally determines the performance indicators of the UAV. Its anterior distance is $400 \mathrm{~km}$, the stable working height is $9000 \mathrm{~m}$, the maximum level flight speed is $670 \mathrm{~km} / \mathrm{h}$, and flying time in the mission area is more than 6 hours. It provides a reference for the future test of UAV.
\end{abstract}

\section{Introduction}

The shipboard alert UAV focuses on the task of ensuring the aircraft carrier formation to perform mid-to-long sea combat missions. It mainly performs reconnaissance and alert missions to make up or enhance the early alert detection capability of aircraft carrier formations. For catapult takeoff aircraft carrier, alert UAVs can be deployed in the secondary threat direction, and with the use of carrier-based fixed-wing air early warning (AEW). For aircraft carriers that using ski-jump take-off, the alert UAVs can be used with carrier-based alert helicopters to deploy on the periphery of the helicopters. The task of carrier-based alert UAVs is the same as or similar to the carrier-based fixed-wing AEW, so we can put forward the technical specifications from the analysis of the performance characteristics of AEW which provide a reference for the future test of UAV and lay the foundation for the research on the overall performance and thermal cycling parameters of the turbofan engine used in shipboard UAVs.

\section{Performance characteristics of carrier-based fixed wing AEW}

The main combat mission of carrier-based fixed-wing AEW is to detect and monitor targets from air or sea, to command and guide aircraft to carry out combat missions at the same time. AEW equipped with high-power radar and special electronic equipment, can perform surveillance and guidance missions. It can also reconnoitre enemy outside the enemy air defense area.

* Correspond author: Lightoffreedom@163.com 
In the development of carrier-based fixed-wing AEW, E-2 was the most successful carrier-based AEW. The aircraft has strong maritime surveillance ability. It usually patrols at a height of $9144 \mathrm{~m}$ and an attack angle of $3^{\circ}$ when performing surveillance missions. It can process hundreds of the tracks and guide intercept dozens batches of aircraft at the same time[1], the main performance indicators was shown in Table 1.

Table 1. The main performance of E-2C.

\begin{tabular}{|c|c|}
\hline Main performance & Parameter \\
\hline Maximum takeoff weight & $24687 \mathrm{~kg}$ \\
\hline Maximum level flying speed & $626 \mathrm{~km} / \mathrm{h}$ \\
\hline Maximum cruising speed & $602 \mathrm{~km} / \mathrm{h}$ \\
\hline Remote cruise speed & $480 \mathrm{~km} / \mathrm{h}$ \\
\hline Service ceiling & $11278 \mathrm{~m}$ \\
\hline Duration of flight & $6.26 \mathrm{~h}$ \\
\hline
\end{tabular}

\section{Analysis of Main Performance Requirements of Shipboard Alert UAVs}

Operational performance and mission of shipboard alert UAV are very similar with the shipboard AEW, its performance parameters and operational modes can refer to the relevant data of shipboard AEW.

In the air defense of aircraft carrier battle formation, its front configuration should be conducive to improving the overall effectiveness of aircraft carrier formation air defense combat system. In order to increase the depth of early warning detection, the activity area should be as far forward as possible. However, the shipboard alert UAV should make the warning range of the shipboard alert UAV coincide with the existing warning range of the aircraft carrier formation, to form a full depth air early warning detection system.

\subsection{Anterior distance analysis}

According to the anterior distance mathematical model of warning helicopter established by the related research, when the helicopter airborne radar detection range of $150 \mathrm{~km}$ and three generations of aircraft flight speed of $1000 \mathrm{~km} / \mathrm{h}$, its anterior distance is between $180 \sim 200$ $\mathrm{km}[2]$. The average anterior distance for the shipboard alert helicopter of 'Sea King' is 185 $\mathrm{km}$ [3], so the anterior distance of alert helicopter is taken at $200 \mathrm{~km}$. In order to maintain early warning detection fan angle of the existing aircraft carrier formations, as shown in figure 1, the anterior distance of shipboard alert UAV should be:

$$
\sin (\theta) \leq r_{1} / d_{1} \leq r_{2} / d_{2}
$$

where $\theta$ is warning detect fan angle, $r_{1}, r_{2}$ are detection distance of shipboard alert helicopter and shipboard alert UAV respectively, $d_{1}, d_{2}$ are anterior distance of the alert helicopter and shipboard alert UAV respectively. 


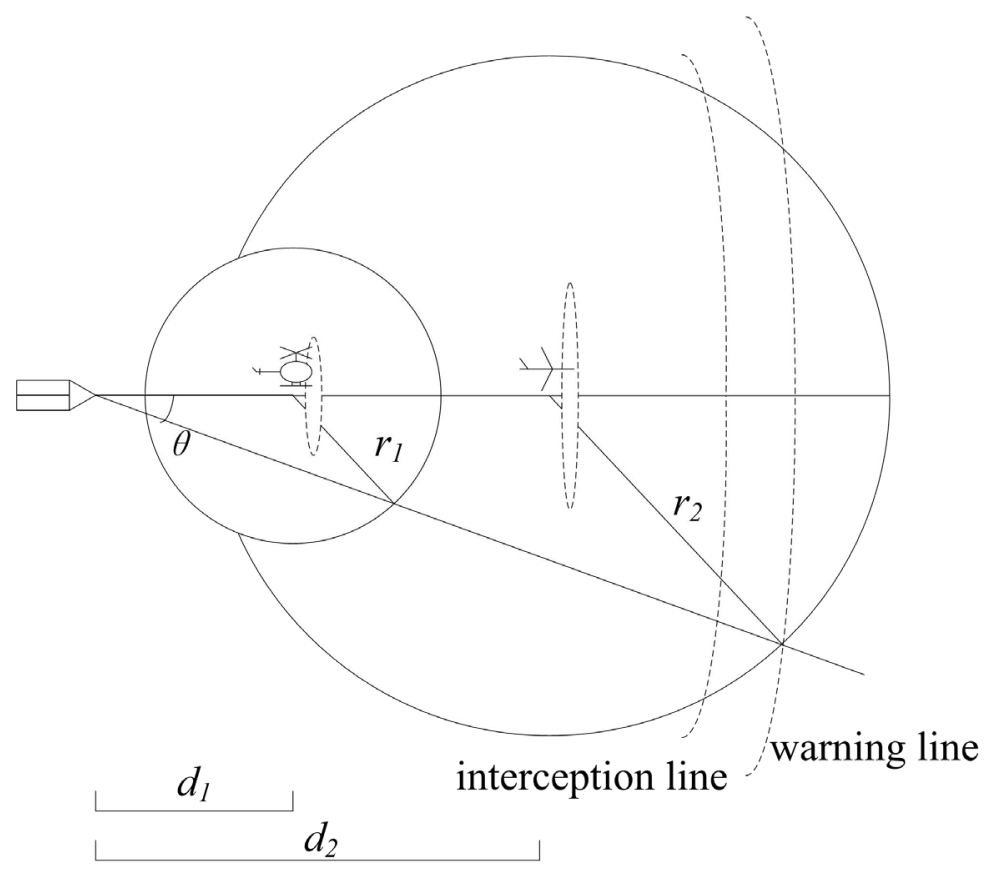

Fig. 1. The anterior distance of UAV.

The low-altitude fighter and low-altitude cruise missiles were found by the radar on shipboard fixed-wing AEW at $410 \mathrm{~km}$ and $300 \mathrm{~km}$ respectively[4]. Without reducing the fan angle of early warning detection, according to equation (1), the anterior distance of the shipboard alert UAV should not exceed $400 \mathrm{~km}$. And the fixed-wing AEW is usually deployed $400 \mathrm{~km}$ before the aircraft carrier formation to perform a task, it is the same distance. If the anterior distance of shipboard alert UAV takes $400 \mathrm{~km}$, the warning distance to low-altitude fighter is $810 \mathrm{~km}$ and the warning distance to low-altitude cruise missiles is $700 \mathrm{~km}$.

\subsection{Working height analysis}

For the detection of cruise missiles and other low-altitude targets, the curvature of the earth restricts the detection range. On the other hand, it is very difficult to detect low-altitude targets from surface clutter which is in the blind spot of air defense radar. For low-altitude targets, the height of the radar determines the radar's limit detection range. The flight altitude of UAV during its mission is usually determined by the mission requirements and the performance of the airborne equipment. At the same time, the effective detection area of airborne radar must cover the area required for combat missions. The effective detection area mainly considers the effective detection range of airborne radar and the direct viewing distance from target affected by the curvature of the earth. The distance between the radar and the target is equal to the geometric direct viewing distance, the geometric distance of the radar is $R_{d}=4.12\left(\sqrt{h_{a}}+\sqrt{h_{t}}\right)$. And the shaded area is the radar blind spot, it is mainly affected by the height of the radar[5] as shown in Figure 2. 


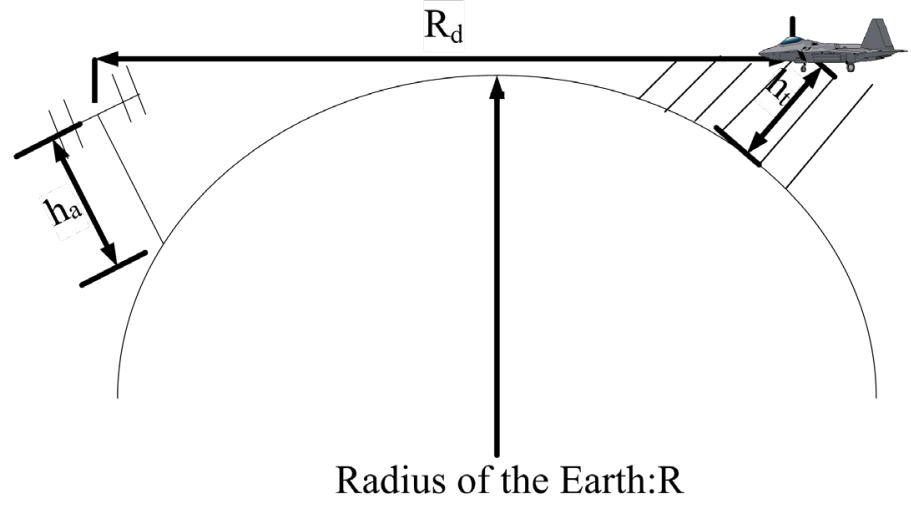

Fig. 2. The visual distance of radar.

If the distance UAV detecting the three generations aircraft was $350 \mathrm{~km}$ and the length of the airborne transmitting antenna was $10 \mathrm{~m}$ when the third generation of enemy aircraft attacked at low altitude, the flight height is at least $6689 \mathrm{~m}$ when performing an early warning mission based on a geometric direct view formula. The adequate visible transmission distance between the shipboard control station and the alert UAV is required to ensure transmission bandwidth and reliability. If the anterior distance of UAV was 400 $\mathrm{km}$ and communication antenna of UAV was $9 \mathrm{~m}$, and the flight height of UAV in the mission area was $8852 \mathrm{~m}$ at least. A typical AEW cruised at a height of $9000 \mathrm{~m}$, and the E$2 \mathrm{C}$ usually flew at a height of $9144 \mathrm{~m}$ with $3^{\circ}$ angle of attack during missions. Considering the above situation and keeping a certain margin, the stability working height of alert UAV should be more than $9000 \mathrm{~m}$.

\subsection{Flight speed analysis}

For the shipboard alert UAV, it is very important to reach the mission area and evacuate the dangerous areas quickly, which requires a faster flying speed. If the enemy fighter was detected by the UAV when it was coming to attack the aircraft carrier formation, UAV began to evacuate and the fighter on the aircraft carrier deck began to take off to intercept enemy. To ensure that enemy airplanes were shot down before launching their air-to-air missiles, it must be satisfied:

$$
\frac{S_{t c}+S_{j l}-D_{d}-V_{d j} t_{y}}{V_{d j}+V_{j}}+t_{g j}+t_{z b}<\frac{S_{t c}-D_{d}-V_{d j} t_{y}}{V_{d j}-V_{w r}}
$$

where $S_{t c}$ was detection range of UAV which takes $350 \mathrm{~km}, S_{j l}$ was anterior distance of UAV which takes $400 \mathrm{~km}, D_{d}$ was range of air to air missile which takes $120 \mathrm{~km}, V_{j}, V_{d j}$ were carrier-based aircraft speed of ours and the enemy which take $1000 \mathrm{~km} / \mathrm{h}, t_{y}$ was the time of alert UAV turn to avoid which takes $2 \mathrm{~min}, t_{z b}$ was the preparation time of shipboard aircraft taking off from carrier deck which takes $15 \mathrm{~min}, t_{g j}$ was the time from launching missiles to hit the target which takes $1.5 \mathrm{~min}$.

In order to ensure that an effective interception can be carried out before an enemy aircraft launches an air-to-air missile on an alert UAV, the flight speed of the UAV should not be less than $671.3 \mathrm{~km} / \mathrm{h}$. The maximum level flight speed of the E-2C is $626 \mathrm{~km} / \mathrm{h}$, and 
the calculated speed is almost same, so the maximum level flight speed of alert UAV is 670 $\mathrm{km} / \mathrm{h}$.

\subsection{Flying time analysis}

Shipboard alert UAV should have a strong ability to endurance, so as to ensure that there are UAVs to carry out the guarding task in the key directions to protect aircraft carrier formation from attack by enemy aircraft during 24 hours. Anterior time $T_{c}$ and return time $T_{f}$ of UAV each needs 1 hour, the preparation time $T_{z}$ needs 1 hour, flying time in the mission area $T_{d}$ should meet $T_{d}>T_{c}+T_{z}+T_{f}$, so $T_{d}$ should be at least 3 hours to ensure that two UAVs rotation to achieve 24-hour vigilance in one direction, as shown in figure 3. If two UAVs were deployed in one direction and the difference between the scheduled time of departure for the two UAVs is three hours, so the minimum $T_{d}$ should be at least six hours to ensure a rotation of three UAVs and 24-hour vigilance. So flying time in the mission area of alert UAVs should be not less than six hours.

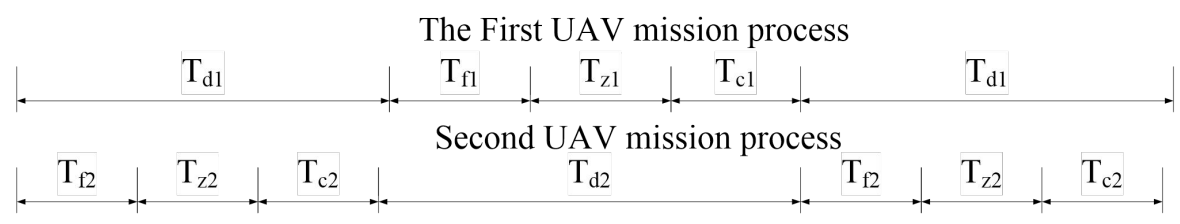

Fig. 3. UAV take turns.

\section{Conclusion}

This paper first analyzes and summarizes the tactical and technical indicators of the carrierbased AEW, then analyzes the tactical indicators of the UAV refer to technical performance of AEW, and finally determines the performance indicators of the UAV. It provides a reference for the future test of UAV and lays the foundation for the research on the overall performance and thermal cycling parameters of the turbofan engine used in shipboard UAVs.

\section{Acknowledgments}

This work is supported by special funds of Taishan Scholar Project, National Natural Science Fund (51505492) and Natural Science Foundation of Shandong Province (ZR2016FQ19).

\section{References}

1. Li S Z and Luo T S, E-2 Series Shipboard Early Warning Aircraft (Beijing: Aviation Industry Press)pp 89-91, 135-42, 207-13 (2013)

2. Zhang D X and Liao X D, Analysis on the Air Defense Combat Capability of American Aircraft Carrier Formation (Air Navigation Missile vol 8) pp 60-65 (2010)

3. Liu T H Kong T and Xie Y H, A Constraint Analysis on Pre-warning Longitudinal Depth of Alert Aircraft Helicopters( Naval Aeronautical Engineering Institute, vol 30) pp 392-95 (2015) 
4. Xu C X, Shipborne AWACS Radar (Modern Ship, vol 8) pp 36-37 (2003)

5. Li J J Wang P and Yin B et al, Efficacy analysis of shipboard helicopters in major combat missions (Ship Electronic Engineering, vol 27) pp 60-62 (2007) 